\title{
Amphiphilic Calcium Alginate Carbon Aerogels: Broad-Spectrum Adsorbents for Ionic and Solvent Dyes with Multiple Functions for Decolorized Oil-Water Separation
}

Xiuxiu Tian ${ }^{\dagger \S}$, Haishan $Z_{\text {hu }}^{\dagger \S}$, Xiao Meng ${ }^{\dagger \S}$, Jiao Wang ${ }^{\dagger \S}$, Chenglin Zheng ${ }^{\S}$, Yanzhi $\mathrm{Xia}^{\S^{*}}$ and Zhong Xiong ${ }^{\dagger \S^{*}}$

$†$ College of Chemistry and Chemical Engineering, Qingdao University, Qingdao 266071, China.

$\S$ Institute of Marine Biobased Materials, Shandong Collaborative Innovation Center of Marine Biobased Fibers and Ecological textiles, State Key Laboratory of Bio-fibers and Eco-textiles, Qingdao University, Qingdao 266071, China.

$\perp$ College of materials science and engineering, Qingdao University, Qingdao 266071, China

* Corresponding Author. E-mail: xiongzhong22@163.com

E-mail: xiayz@qdu.edu.cn

Total Pages: 9

Total number of Figures: 6

Total number of Table: 4 


\section{Contents}

Figure S1. Chemical structures of all the dyes utilized in this study. Cationic dyes: MB, MG, CV, and RB; anionic dyes: MO, AF, SY, and CR; solvent dyes: oil red O MVB, SO, and FY.

Figure S2. TGA curve of CA.

Figure S3. High-resolution (a) O 1s and (b) Ca 2p XPS spectra of CCA-1000.

Figure S4. High-resolution (a) C 1s, (b) O 1s, and (c) Ca 2p XPS spectra of CCA-800.

Figure S5. The adsorption process of CCA-500 and CA for MB, MO, and oil red O.

Figure S6. UV-vis absorptions of dye solutions before and after decolorization after 7 h by CA, CCA-500, CCA-800, and CCA-1000. (a) MB, (b) MO, and (c) oil red $\mathrm{O}$.

Table S1 Peak positions and areas of CCA-800 and CCA-1000 calculated by XPS results.

Table S2 Kinetic parameters for the adsorption of $\mathrm{MB}, \mathrm{MO}$, and oil red $\mathrm{O}$ on CCA-1000.

Table S3 Thermodynamic parameters for the adsorption of MB, MO, and oil red O on CCA-1000.

Table S4 The reported adsorption capacities of various adsorbents for MG, CV, and AF dye with the result of our work. 
<smiles>CN(C)c1ccc2nc3ccc(=[N+](C)C)cc-3sc2c1</smiles>

Methylene blue<smiles>CN(C)c1ccc(C(=C2C=CC(=[N+](C)C)C=C2)c2ccccc2)cc1</smiles>

Malachite green oxalate<smiles>CN(C)c1ccc(N=Nc2ccc(S(=O)(=O)[O-])cc2)cc1</smiles>

Methyl orange<smiles>Cc1ccc(C)c(/N=N/c2cc(C)c(/N=N\c3c(O)ccc4ccccc34)cc2C)c1</smiles>

Oil red $\mathrm{O}$<smiles>CCN(CC)c1ccc2c(-c3ccccc3C(=O)O)c3ccc(=[N+](CC)CC)cc-3oc2c1</smiles>

Rhodamine B<smiles>Cc1cc(C(=C2C=CC(=N)C(S(=O)(=O)O)=C2)c2ccc(N)c(S(C)(=O)=O)c2)cc(S(=O)(=O)O)c1N</smiles>

Acid fuchsin<smiles>CN=C1C=CC(=C(c2ccc(N(C)C)cc2)c2ccc(N(C)C)cc2)C=C1</smiles>

Methyl violet B base<smiles>CN(C)c1ccc(C(c2ccc(N(C)C)cc2)C2C=CC(=[N+](C)C)C=C2)cc1</smiles>

Crystal violet<smiles>Nc1c(N=Nc2ccc(-c3ccc(N=Nc4cc(S(=O)(=O)O[Na])c5ccccc5c4N)cc3)cc2)cc(S(=O)(=O)O[Na])c2ccccc12</smiles>

Sunset yellow

\section{Congo red}

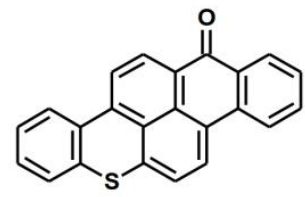

Solvent orange 63

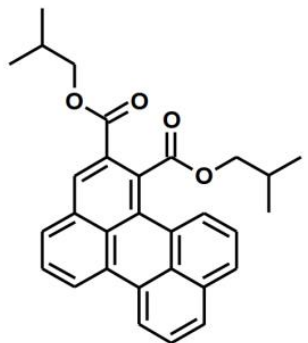

Fluorescent yellow $8 \mathrm{~g}$

Figure S1. Chemical structures of all the dyes utilized in this study. Cationic dyes:

MB, MG, CV, and RB; anionic dyes: MO, AF, SY, and CR; solvent dyes: oil red O MVB, SO, and FY. 


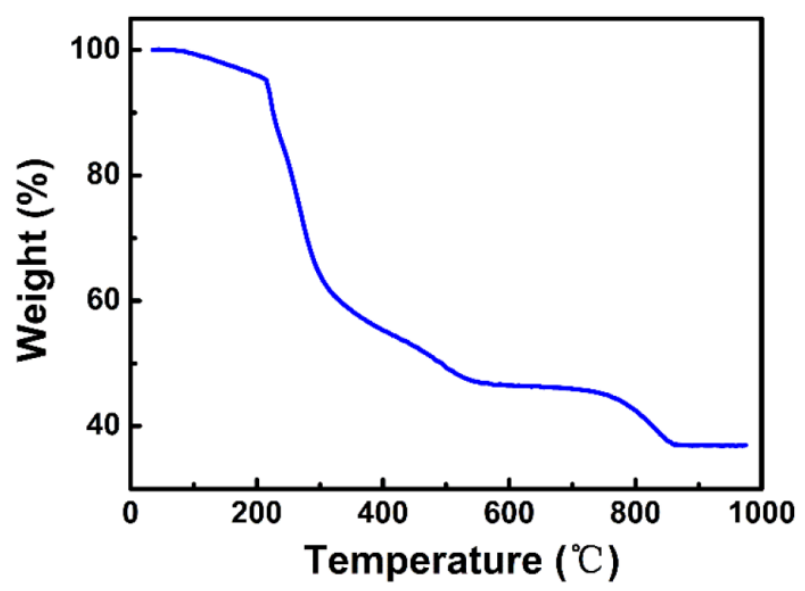

Figure S2. TGA curve of CA
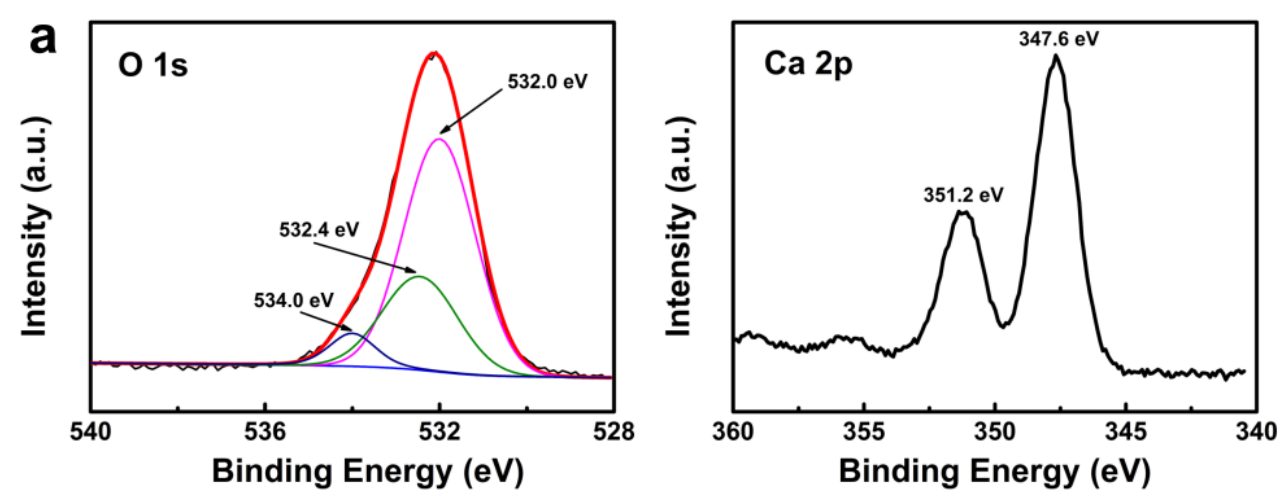

Figure S3. High-resolution (a) O 1s and (b) Ca 2p XPS spectra of CCA-1000.
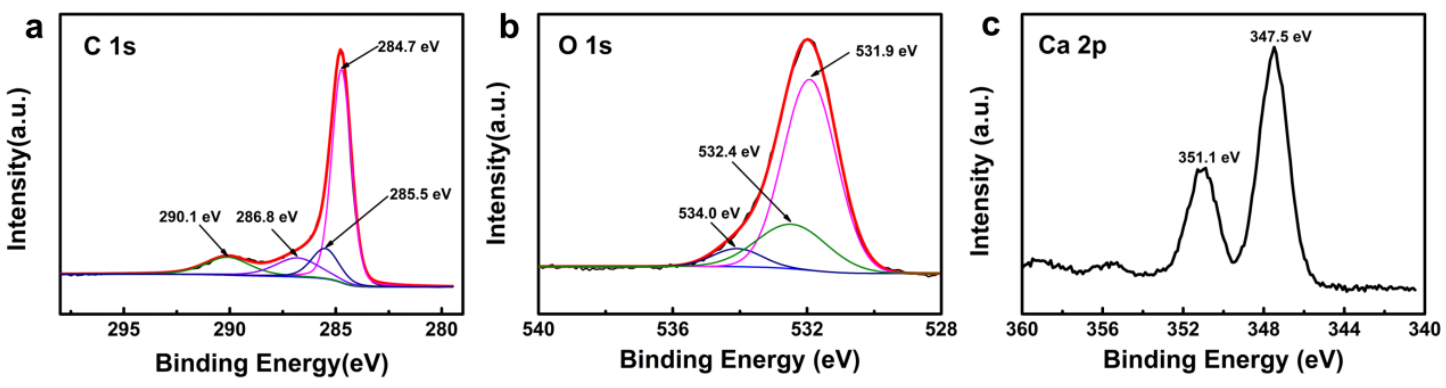

Figure S4. High-resolution (a) C 1s, (b) O 1s, and (c) Ca 2p XPS spectra of CCA-800. 


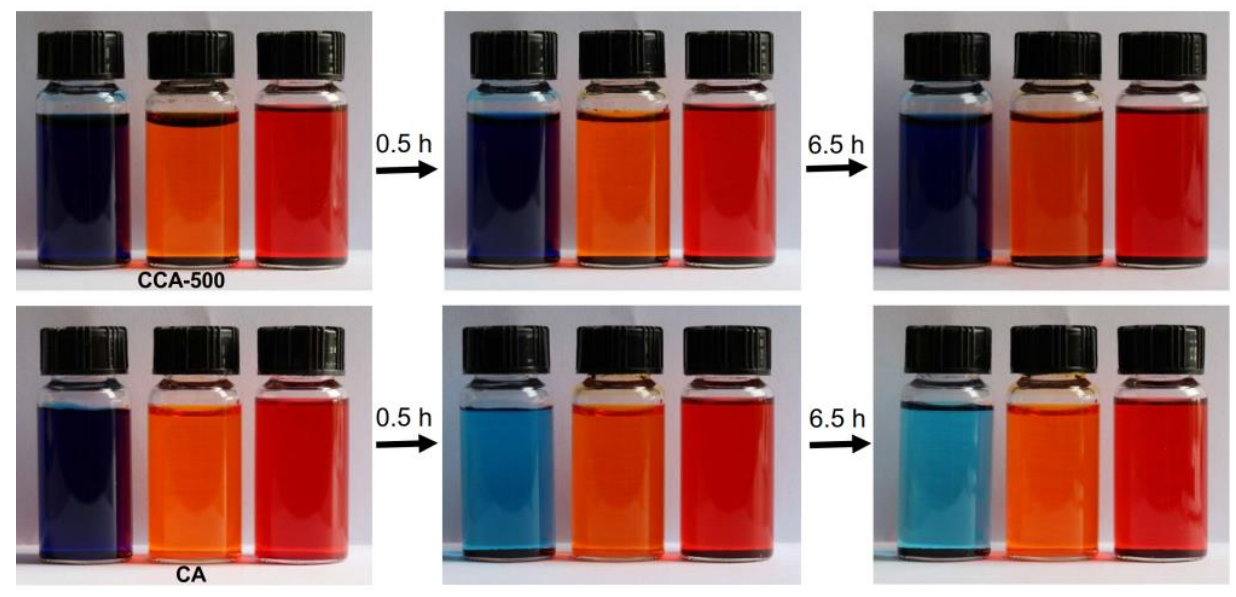

Figure S5. The adsorption process of CCA-500 and CA for MB, MO, and oil red O.
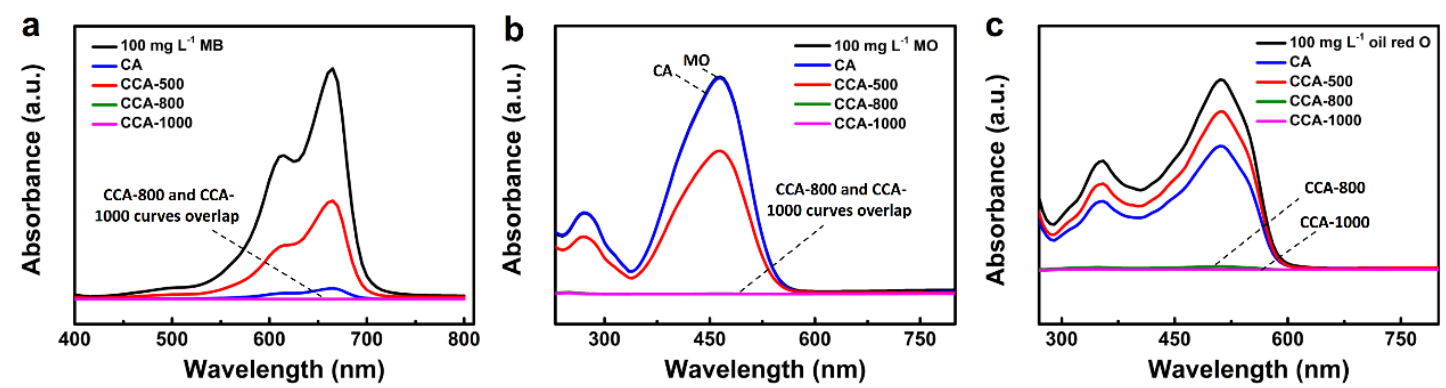

Figure S6. UV-vis absorptions of dye solutions before and after decolorization after 7

h by CA, CCA-500, CCA-800, and CCA-1000. (a) MB, (b) MO, and (c) oil red O.

Table S1 Peak positions and areas of CCA-800 and CCA-1000 calculated by XPS results.

\begin{tabular}{lllllllll}
\hline Samples & & \multicolumn{3}{c}{$\mathrm{C}$} & \multicolumn{3}{c}{$\mathrm{O}$} \\
\cline { 3 - 8 } & & $\mathrm{C}=\mathrm{C}$ & $\mathrm{C}-\mathrm{C}$ & $\mathrm{C}-\mathrm{O}$ & $\pi-\pi$ & $\mathrm{C}-\mathrm{O}$ & $\mathrm{CaO}$ & $\mathrm{H}_{2} \mathrm{O}$ \\
\hline CCA-800 & Peak & 284.75 & 285.5 & 286.8 & 290.1 & 531.92 & 532.4 & 534.08 \\
& $\begin{array}{l}\text { position } \\
\text { Area }\end{array}$ & 28634.6 & 6101.96 & 6318.5 & 6258.26 & 20179.6 & 6187.8 & 1754 \\
CCA-1000 & Peak & 284.73 & 285.79 & 286.8 & 290.1 & 532.0 & 532.4 & 534.0 \\
& position & & & & & & & \\
& Area & 31040.6 & 4941.92 & 5913.2 & 7371.1 & 19581.3 & 8304.7 & 2024.1 \\
\hline
\end{tabular}


Table S2 Kinetic parameters for the adsorption of MB, MO, and

$$
\text { oil red O on CCA-1000 }
$$

\begin{tabular}{ccccccc}
\hline Dyes & \multicolumn{3}{c}{ Pseudo-first-order model } & \multicolumn{3}{c}{ Pseudo-second-order model } \\
\hline & $\mathrm{K}_{1}\left(\mathrm{~min}^{-1}\right)$ & $\mathrm{Q}_{\mathrm{e}, \mathrm{cal}}(\mathrm{mg} / \mathrm{g})$ & $\mathrm{R}^{2}$ & $\mathrm{~K}_{2}$ & $\mathrm{Q}_{\mathrm{e}, \mathrm{cal}}(\mathrm{mg} / \mathrm{g})$ & $\mathrm{R}^{2}$ \\
$\mathrm{MB}$ & 0.00546 & 193.72 & 0.9921 & 0.0048 & 203.62 & 0.99993 \\
MO & 0.00233 & 103.88 & 0.1165 & 0.00943 & 108.43 & 0.99988 \\
Oil red O & 0.00385 & 64.80 & 0.94141 & 0.01473 & 67.09 & 0.99993 \\
\hline
\end{tabular}

Table S3 Thermodynamic parameters for the adsorption of MB, MO, and oil red O on CCA-1000

\begin{tabular}{lcllllll}
\hline \multirow{2}{*}{ Dyes } & \multirow{2}{*}{$\Delta H(\mathrm{~kJ} / \mathrm{mol})$} & $\Delta S(\mathrm{~J} / \mathrm{mol} / \mathrm{K})$ & \multicolumn{5}{c}{$\Delta G(\mathrm{~kJ} / \mathrm{mol})$} \\
\cline { 4 - 7 } & & & $298 \mathrm{~K}$ & $308 \mathrm{~K} 318 \mathrm{~K}$ & $328 \mathrm{~K}$ & $338 \mathrm{~K}$ \\
\hline MB & $23.74 /-51.25$ & $96.08 /-139.50$ & -4.89 & -5.85 & -6.81 & -5.49 & -4.09 \\
MO & -11.24 & -26.63 & -3.30 & -3.04 & -2.77 & -2.50 & -2.24 \\
Oil red O & 2.84 & 22.22 & -3.78 & -3.99 & -4.22 & -4.44 & -4.66 \\
\hline
\end{tabular}

Table S4 The reported adsorption capacities of various adsorbents for MG, CV, and AF dye with the result of our work.

\begin{tabular}{llll}
\hline Adsorbates & Adsorbents & $\begin{array}{l}\text { Adsorption } \\
\text { capacity }(\mathrm{mg} \\
\left.\mathrm{g}^{-1}\right)\end{array}$ & Ref. \\
\hline $\mathrm{MG}$ & Calcium-rich biochar (CRB) & 12502 & {$[1]$} \\
& Chromium doped zinc oxide nanoparticles & 214.0 & {$[2]$} \\
& KOH-activated sodium carboxymethyl cellulose & 245.3 & {$[3]$} \\
& (CMC) carbon aerogels & & \\
Exfoliated graphite (EG) & 384.6 & {$[4]$} \\
& Tetraethylenepentamine-functionalized Rosa & 333.3 & {$[5]$} \\
& canina-L fruits activated carbon (TEPFRCA) & &
\end{tabular}


Acetylated sodium 2,3-dicarboxyhemicellulose

456.23

Reduced graphene oxide (rGO).

476.2

Calcium alginate carbon aerogels

7059

CV

Porous 3D rGO/ZIF-67 aerogel

1714.2

Our work

Biopolymer/ZSM-5 zeolite

1217.3

[8]

Starch-derived carbon aerogels

1515

$\alpha-\mathrm{MnO}_{2}$ microspheres

5882.3

Calcium alginate carbon aerogels

Polymer-derived mesoporous $\mathrm{Ni} / \mathrm{SiOC}(\mathrm{H})$

129.8

$\mathrm{ZnO}$ nanoparticles

Polypyrrole/CNTs- $\mathrm{CoFe}_{2} \mathrm{O}_{4}$ magnetic nanohybrid

132.15

Carboxymethyl-chitosan/bentonite composite

Ammonium-functionalized MCM-41

\section{References}

(1) Dai, L.; Zhu, W.; He, L.; Tan, F.; Zhu, N.; Zhou, Q.; He, M.; Hu, G. Calcium-rich biochar from crab shell: An unexpected super adsorbent for dye removal. Bioresource Technology 2018, 267, 510-516.

(2) Jamshidi, M.; Ghaedi, M.; Dashtian, K.; Hajati, S.; Bazrafshan, A. A. Sonochemical assisted hydrothermal synthesis of $\mathrm{ZnO}$ : $\mathrm{Cr}$ nanoparticles loaded activated carbon for simultaneous ultrasound-assisted adsorption of ternary toxic organic dye: Derivative spectrophotometric, optimization, kinetic and isotherm study. Ultrason. Sonochem. 2016, 32, 119-131.

(3) Yu, M.; Li, J.; Wang, L. KOH-activated carbon aerogels derived from sodium carboxymethyl cellulose for high-performance supercapacitors and dye adsorption. Chem. Eng. J. 2017, 310, 300-306. 
(4) Sykam, N.; Jayram, N. D.; Rao, G. M. Highly efficient removal of toxic organic dyes, chemical solvents and oils by mesoporous exfoliated graphite: Synthesis and mechanism. Journal of Water Process Engineering 2018, 25, 128-137.

(5) Ghasemi, M.; Mashhadi, S.; Asif, M.; Tyagi, I.; Agarwal, S.; Gupta, V. K. Microwave-assisted synthesis of tetraethylenepentamine functionalized activated carbon with high adsorption capacity for Malachite green dye. J. Mol. Liq. 2016, $213,317-325$.

(6) Gautam, D.; Kumari, S.; Ram, B.; Chauhan, G. S.; Chauhan, K. A new hemicellulose-based adsorbent for malachite green. J. Environ. Chem. Eng. 2018, 6, 3889-3897.

(7) Gupta, K.; Khatri, O. P. Reduced graphene oxide as an effective adsorbent for removal of malachite green dye: Plausible adsorption pathways. J. Colloid Interf. Sci. 2017, 501, 11-21.

(8) Yang, Q.; Lu, R.; Ren, S.; Chen, C.; Chen, Z.; Yang, X. Three dimensional reduced graphene oxide/ZIF-67 aerogel: Effective removal cationic and anionic dyes from water. Chem. Eng. J. 2018, 348, 202-211.

(9) Brião, G. V.; Jahn, S. L.; Foletto, E. L.; Dotto, G. L. Highly efficient and reusable mesoporous zeolite synthetized from a biopolymer for cationic dyes adsorption. Colloids Surf. A 2018, 556, 43-50.

(10) Chang, X.; Chen, D.; Jiao, X. Starch-derived carbon aerogels with high-performance for sorption of cationic dyes. Polymer 2010, 51, 3801-3807. 
(11) Abraham, R.; Mathew, S.; Kurian, S.; Saravanakumar, M. P.; Ealias, A. M.; George, G. Facile synthesis, growth process, characterisation of a nanourchin-structured $\alpha-\mathrm{MnO}_{2}$ and their application on ultrasonic-assisted adsorptive removal of cationic dyes: A half-life and half-capacity concentration approach. Ultrason. Sonochem. 2018, 49, 175-189.

(12) Yu, Z.; Li, S.; Zhang, P.; Feng, Y.; Liu, X. Polymer-derived mesoporous $\mathrm{Ni} / \mathrm{SiOC}(\mathrm{H})$ ceramic nanocomposites for efficient removal of acid fuchsin. Ceram. Int. 2017, 43, 4520-4526.

(13) Zhang, F.; Chen, X.; Wu, F.; Ji, Y. High adsorption capability and selectivity of $\mathrm{ZnO}$ nanoparticles for dye removal. Colloids Surf. A 2016, 509, 474-483.

(14) Li, X.; Lu, H.; Zhang, Y.; He, F. Efficient removal of organic pollutants from aqueous media using newly synthesized polypyrrole/CNTs-CoFe ${ }_{2} \mathrm{O}_{4}$ magnetic nanocomposites. Chem. Eng. J. 2017, 316, 893-902.

(15) Gong, N.; Liu, Y.; Huang, R. Simultaneous adsorption of $\mathrm{Cu}^{2+}$ and Acid fuchsin (AF) from aqueous solutions by $\mathrm{CMC} /$ bentonite composite. Int. J. Biol. Macromol. 2018,115, 580-589.

(16) Qin, Q.; Ma, J.; Liu, K. Adsorption of anionic dyes on ammonium-functionalized MCM-41. J. Hazard. Mater. 2009, 162, 133-139. 